\title{
Calculation of the Impedance of an E-cored Coil Placed Above a Conductive Material with a Surface Hole
}

\author{
Grzegorz Tytko, Leszek Dziczkowski \\ Institute of Electronics, Faculty of Automatic Control, Electronics and Computer Science, The Silesian University of \\ Technology, Akademicka 16, 44-100, Gliwice, Poland, grzegorz.tytko@polsl.pl
}

\begin{abstract}
In eddy current testing, probe-coils with the E-type pot core are commonly used for detecting defects in test objects. In this paper, an analytical mathematical model of such a probe placed above a two-layered conductive material with a surface hole has been presented. The final formulas in the closed form that make it possible to calculate the coil impedance were worked out using the truncated region eigenfunction expansion (TREE) method and implemented in Matlab. Changes in resistance and reactance were determined for both material without a hole and for space containing no conductor. The results were compared with those obtained for the air-cored coil and the I-cored coil. The correctness of the calculations was confirmed through experimental measurements and with the finite element method (FEM) in the COMSOL Multiphysics package.
\end{abstract}

Keywords: Nondestructive testing, eddy current, coil impedance, ferrite core, analytical model, truncated region eigenfunction expansion.

\section{INTRODUCTION}

One of the most frequently employed methods of detecting surface flaws in conductive materials is the eddy current technique. In this method, the probe in the form of a coil fed with alternating current is placed above the test object in which eddy current induction takes place. All the defects occurring in the conductor disturb the flow of eddy currents, which results in changes in the impedance of the coil. The correct interpretation of these changes allows effective detection of defects in materials. What serves this purpose is the employment of appropriate mathematical models that enable simulation of the measurement process and calculation of the changes in the coil impedance.

The probe-coil placed above the test object with a flaw was analyzed by applying the pulsed eddy current technique (PEC) [1]-[3] with the employment of the grating eddy current displacement sensor (GECDS) [4], and considered as the inverse problem [5], [6]. The analytical mathematical models of a coil brought closer to conductive material with a hole were obtained for air-cored coils [7]-[9] and I-cored coils [10], [11] using the truncated region eigenfunction expansion (TREE) method. In eddy current testing, however, the most commonly used probes are pot core probes. The closing of the magnetic flux in the interior of such a core facilitates obtaining greater sensitivity of the probe and, consequently, its higher efficiency in detecting flaws. Unfortunately, the analytical models of pot core probes developed previously [12], [13] do not take into account the presence of defect in the test object, which makes it impossible to use them in the eddy current defectoscopy. What is the missing solution is the analytical model of the E-cored coil, proposed in the paper, placed above a two-layered conductive material with a surface hole (Fig.1.). It allows calculation of both the impedance of the coil and its changes caused by the presence of the hole. The presented solution was worked out using the TREE method, and implemented in Matlab. The results were verified with the employment of the finite element method (FEM) and through experiments. In all the cases, a very good agreement was observed.

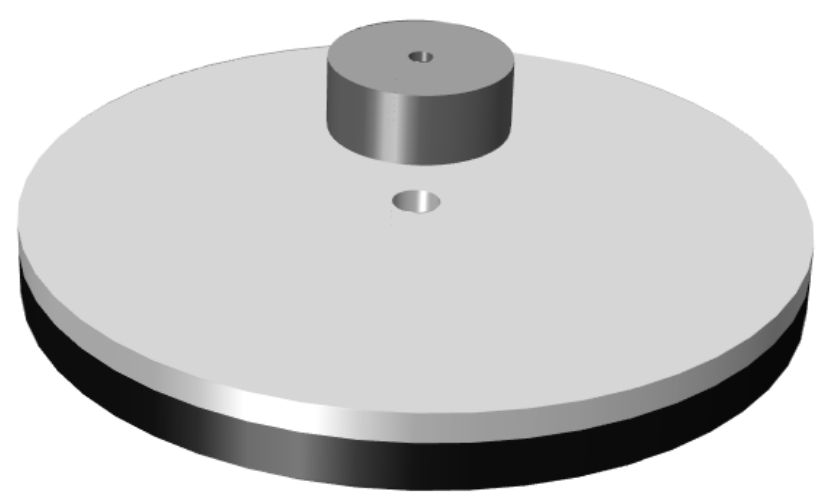

Fig.1. E-cored coil located above a conductive material with a surface hole. 


\section{SOLUTION}

The problem of the E-cored coil placed above the twolayered conductive material of relative permeability $\mu_{6}, \mu_{7}$ and electrical conductivity $\sigma_{6}, \sigma_{7}$ (Fig.2.) was solved using the TREE method. The domain of the solution was limited in the radial direction to the value of the parameter $b$. The vanishing of the magnetic vector potential $A_{\varphi}(r, z)=0$ for the internal $(r=0)$ and external $(r=b)$ boundary of the domain was ensured by satisfying the homogeneous Dirichlet condition. The system was divided into 8 regions and 5 sub-regions $(\mathrm{I}-\mathrm{V})$. The determination of the magnetic vector potential for each of the regions required calculating discrete eigenvalues. For the regions composed only of air $(1,5)$, discrete eigenvalues $q_{\mathrm{i}}$ were obtained from the equation

$$
J_{1}\left(q_{\mathrm{i}} b\right)=0,
$$

where $J_{1}(x)$ is the Bessel function of the first kind. The expressions that allow calculating the eigenvalues $m_{\mathrm{i}}$ of region 2 and eigenvalues $p_{\mathrm{i}}$ of regions 3, 3-4, 4 have been presented in [10] and [13], respectively. The material coefficients $S_{7 \mathrm{i}}$ of region 7, which depend on the frequency excitation $\omega$, the relative magnetic permeability $\mu_{7}$, the permeability of free space $\mu_{0}$ and the electrical conductivity $\sigma_{7}$, were calculated using the value of $q_{\mathrm{i}}$ in accordance with the expression:

$$
s_{7 \mathrm{i}}=\sqrt{q_{\mathrm{i}}^{2}+j \omega \mu_{7} \mu_{0} \sigma_{7}}
$$

In the surface layer of the test material there is a hole with radius $g$ and depth $l_{2}-l_{1}$. Its presence results in the fact that the eigenvalues $u_{\mathrm{i}}$ of region 6 are roots of the complex function that satisfies the electromagnetic interface conditions between the hole and the conductive material [10], [11], [14], [15].

$$
u_{\mathrm{i}} P_{1}\left(v_{\mathrm{i}} g\right) J_{0}\left(u_{\mathrm{i}} g\right)=\frac{1}{\mu_{6}} v_{\mathrm{i}} J_{1}\left(u_{\mathrm{i}} g\right) P_{0}\left(v_{\mathrm{i}} g\right),
$$

where $Y_{\mathrm{n}}(x)$ is the Bessel function of the second kind of order $n, v_{\mathrm{i}}=\left(u_{\mathrm{i}}{ }^{2}-j \omega \mu_{6} \mu_{0} \sigma_{6}\right)^{1 / 2}$, whereas the function $P_{\mathrm{n}}(x)$ has been defined as:

$$
P_{\mathrm{n}}\left(v_{\mathrm{i}} g\right)=Y_{1}\left(v_{\mathrm{i}} b\right) J_{\mathrm{n}}\left(v_{\mathrm{i}} g\right)+J_{1}\left(v_{\mathrm{i}} b\right) Y_{\mathrm{n}}\left(v_{\mathrm{i}} g\right) .
$$

The discrete eigenvalues $u_{\mathrm{i}}$ were calculated through solving (3) with the multilevel computation of the complex eigenvalues (MCCE) method [16]. In this approach, the solution domain is divided into 2 regions. In order to find complex roots, sets of initial points are used in the first of the regions, whereas the Cauchy argument principle is employed in the case of the other region.

Having all eigenvalues $q_{\mathrm{i}}, m_{\mathrm{i}}, p_{\mathrm{i}}, u_{\mathrm{i}}$, and coefficients $s_{7 \mathrm{i}}$ makes it possible to determine the magnetic vector potential for each region. For this purpose, the most convenient is making use of an ideal filamentary coil, whose all turns are placed in a circle of radius $r_{0}$, at a distance $h_{0}$ from the lower edge of the core. In this case, the dimensions of the cylindrical coil shown in Fig.2. are reduced to the formula $r_{2}-r_{1} \rightarrow 0$ and $h_{2}-h_{1} \rightarrow 0$. For the filamentary coil specified in such a way, the magnetic vector potential of each region was written by using matrix notation.

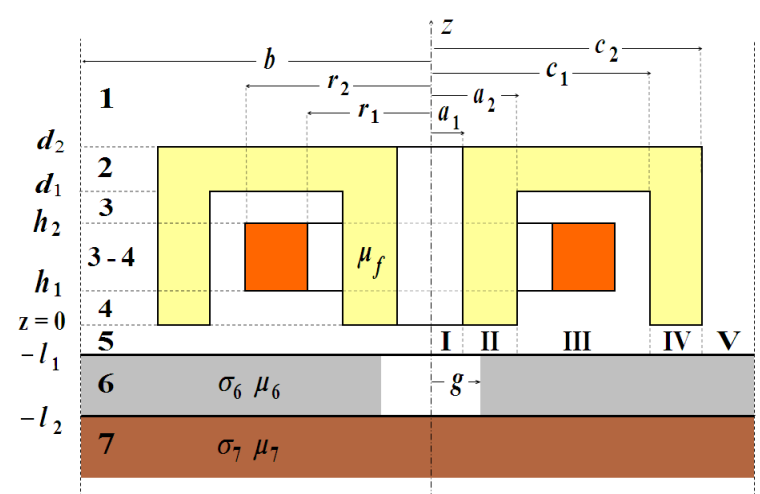

Fig.2. Rectangular cross-sectional coil located above a two-layered conductive material with a surface hole.

$$
\begin{aligned}
& \mathrm{A}_{1}(r, z)=J_{1}\left(\mathbf{q}^{\mathbf{T}} r\right) \mathbf{q}^{-1} e^{-\mathbf{q} z} \mathbf{C}_{1} \\
& J_{1}\left(\mathbf{m}^{\mathrm{T}} r\right) \\
& 0 \leq r \leq a_{1} \\
& \mathrm{~A}_{2}(r, z)=L_{1}\left(\mathbf{m}^{\mathbf{T}} r\right) \mathbf{m}^{-1}\left(e^{-\mathbf{m} z} \mathbf{C}_{2}-e^{\mathbf{m} z} \mathbf{B}_{2}\right) \quad a_{1} \leq r \leq c_{2} \\
& L_{1}^{\prime}\left(\mathbf{m}^{\mathrm{T}} r\right) \\
& c_{2} \leq r \leq b
\end{aligned}
$$

$$
\begin{array}{rlr} 
& J_{1}\left(\mathbf{p}^{\mathbf{T}} r\right) & 0 \leq r \leq a_{1} \\
& R_{1}\left(\mathbf{p}^{\mathbf{T}} r\right) & a_{1} \leq r \leq a_{2} \\
\mathrm{~A}_{3}(r, z)= & R_{1}^{\prime}\left(\mathbf{p}^{\mathbf{T}} r\right) \mathbf{p}^{-1}\left(e^{-\mathbf{p} z} \mathbf{C}_{3}-e^{\mathbf{p} z} \mathbf{B}_{3}\right) & a_{2} \leq r \leq c_{1} \\
& R_{1}^{\prime \prime}\left(\mathbf{p}^{\mathbf{T}} r\right) & c_{1} \leq r \leq c_{2} \\
R_{1}^{\prime \prime \prime}\left(\mathbf{p}^{\mathbf{T}} r\right) & c_{2} \leq r \leq b
\end{array}
$$

$$
\begin{aligned}
& J_{1}\left(\mathbf{p}^{\mathbf{T}} r\right) \\
& R_{1}\left(\mathbf{p}^{\mathbf{T}} r\right) \\
& 0 \leq r \leq a_{1} \\
& a_{1} \leq r \leq a_{2} \\
& \mathrm{~A}_{4}(r, z)=R_{1}^{\prime}\left(\mathbf{p}^{\mathbf{T}} r\right) \mathbf{p}^{-1}\left(e^{-\mathbf{p} z} \mathbf{C}_{4}-e^{\mathbf{p} z} \mathbf{B}_{4}\right) \quad a_{2} \leq r \leq c_{1} \\
& R_{1}^{\prime \prime}\left(\mathbf{p}^{\mathrm{T}} r\right) \\
& c_{1} \leq r \leq c_{2} \\
& R_{1}^{\prime \prime \prime}\left(\mathbf{p}^{\mathbf{T}} r\right) \\
& c_{2} \leq r \leq b \\
& \mathrm{~A}_{6}(r, z)=\begin{array}{ll}
J_{1}\left(\mathbf{u}^{\mathbf{T}} r\right) P_{1}(\mathbf{v} g) \\
P_{1}\left(\mathbf{v}^{\mathbf{T}} r\right) J_{1}(\mathbf{u} g)
\end{array} \mathbf{u}^{-1}\left(e^{-\mathbf{u} z} \mathbf{C}_{6}-e^{\mathbf{u z} z} \mathbf{B}_{6}\right) \begin{array}{l}
0 \leq r \leq g \\
g \leq r \leq b
\end{array}
\end{aligned}
$$

$$
\mathrm{A}_{7}(r, z)=-J_{1}\left(\mathbf{q}^{\mathbf{T}} r\right) \mathbf{s}_{7}^{-1} e^{\mathbf{s}_{7} z} \mathbf{B}_{7}
$$


where

$$
J_{1}\left(\mathbf{q}^{\mathbf{T}} r\right), J_{1}\left(\mathbf{m}^{\mathbf{T}} r\right), J_{1}\left(\mathbf{p}^{\mathbf{T}} r\right), J_{1}\left(\mathbf{u}^{\mathbf{T}} r\right), L_{1}\left(\mathbf{m}^{\mathbf{T}} r\right), R_{1}\left(\mathbf{p}^{\mathbf{T}} r\right), P_{1}\left(\mathbf{v}^{\mathbf{T}} r\right)
$$

are row vectors, $\mathbf{q}, \mathbf{m}, \mathbf{p}, \mathbf{u}, \mathbf{v}, \mathbf{s}_{7}, \mathrm{e}^{\mathbf{x}}$ are diagonal matrices, while $\mathbf{C}_{\mathrm{i}}, \mathbf{B}_{\mathrm{i}}$ are column vectors of unknown coefficients. Using (5) - (11), it is possible to determine the magnetic vector potential of each region of the cylindrical coil (Fig.2.).

$$
A(r, z)=\int_{h_{1}}^{h_{2}} \int_{r_{1}}^{r_{2}} A_{\text {filamentary }}\left(r, z, r_{0}, h_{0}\right) d h_{0} d r_{0}
$$

The expression for the impedance of the E-cored coil was worked out through integrating the magnetic vector potential of region 3-4 over the cross-section of the coil. In this way, the following has been obtained

$$
\begin{aligned}
& Z=\frac{j \omega \pi \mu_{0} N^{2}}{\left[\left(r_{2}-r_{1}\right)\left(h_{2}-h_{1}\right)\right]^{2}} \chi\left(\mathbf{p}^{\mathbf{T}} r_{1}, \mathbf{p}^{\mathbf{T}} r_{2}\right) \mathbf{p}^{-4} \\
& \left\{\left[2\left(h_{2}-h_{1}\right) \mathbf{p}+e^{\mathbf{p}\left(h_{1}-h_{2}\right)}-e^{\mathbf{p}\left(h_{2}-h_{1}\right)}\right]\right. \\
& \left.+\mathbf{W}_{1} \mathbf{W}_{2}^{-1}\left(\boldsymbol{\beta}_{1}-\boldsymbol{\beta}_{2}\right)\right\} \mathbf{p}^{-3} \mathbf{D}^{-1} \chi\left(\mathbf{p} r_{1}, \mathbf{p} r_{2}\right)
\end{aligned}
$$

where

$$
\begin{gathered}
\mathbf{W}_{1}=\left(e^{-\mathbf{p} h_{1}}-e^{-\mathbf{p} h_{2}}\right) \mathbf{C}_{47}-\left(e^{\mathbf{p} h_{2}}-e^{\mathbf{p} h_{1}}\right) \mathbf{B}_{47}, \\
\mathbf{W}_{2}=(\mathbf{T}-\mathbf{U}) e^{-\mathbf{m} d_{2}} \mathbf{C}_{27}-(\mathbf{T}+\mathbf{U}) e^{\mathbf{m} d_{2}} \mathbf{B}_{27}, \\
\boldsymbol{\beta}_{1}=\frac{1}{2}(\mathbf{T} \pm \mathbf{U}) e^{ \pm \mathbf{m} d_{2}} \mathrm{e}^{\mp \mathbf{m} d_{1}} \mathbf{F}^{-1}\left[(\mathbf{H}+\mathbf{G}) e^{ \pm \mathbf{p} d_{1}}\right. \\
\boldsymbol{\beta}_{2} \\
\left.\left(e^{\mp \mathbf{p} h_{1}}-e^{\mp \mathbf{p} h_{2}}\right)+(\mathbf{H}-\mathbf{G}) e^{\mp \mathbf{p} d_{1}}\left(e^{ \pm \mathbf{p} h_{2}}-e^{ \pm \mathbf{p} h_{1}}\right)\right] \\
\chi\left(\mathbf{p} r_{1}, \mathbf{p} r_{2}\right)=\int_{\mathbf{p} r_{1}}^{\mathbf{p} r_{2}} r R_{1}^{\prime}(\mathbf{p} r) d r, \\
\mathbf{B}_{27}=\frac{1}{2} \mathrm{e}^{\mp \mathbf{m} d_{1}} \mathbf{F}^{-1}\left[(\mathbf{H} \mp \mathbf{G}) e^{-\mathbf{p} d_{1}} \mathbf{C}_{47}+(\mathbf{H} \pm \mathbf{G}) e^{\mathbf{p} d_{1}} \mathbf{B}_{47}\right], \\
\mathbf{C}_{27}
\end{gathered}
$$

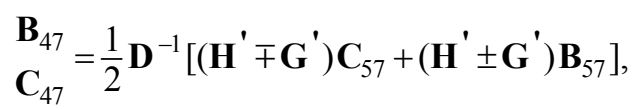

$$
\begin{aligned}
& \mathbf{B}_{57} \\
& \mathbf{C}_{57}
\end{aligned}=\mp \frac{1}{2} e^{ \pm \mathbf{q} l_{1}}\left[\left(\mathbf{u}^{-1} \mathbf{K q} \mp \mathbf{V}\right) e^{\mathbf{u} l_{1}} \mathbf{C}_{67}-\left(\mathbf{u}^{-1} \mathbf{K q} \pm \mathbf{V}\right) e^{-\mathbf{u} l_{1}} \mathbf{B}_{67}\right],
$$

$$
\begin{gathered}
\mathbf{B}_{67}=\frac{1}{2} e^{ \pm \mathbf{u} l_{2}} e^{-\mathbf{s}_{7} l_{2}}\left(\frac{\mathbf{V}^{-1}}{\mu_{7}} \pm \mathbf{s}_{7}^{-1} \mathbf{K}^{-1} \mathbf{u}\right), \\
\mathbf{K}=\left[k_{\mathrm{ij}}\right]=\lambda_{1}+\lambda_{2}, \\
\mathbf{V}=\left[v_{\mathrm{ij}}\right]=\lambda_{1}+\frac{1}{\mu_{6}} \lambda_{2},
\end{gathered}
$$

$$
\lambda_{1}=\frac{g P_{1}\left(v_{\mathrm{j}} g\right)}{u_{\mathrm{j}}^{2}-q_{\mathrm{i}}^{2}}\left[q_{\mathrm{i}} J_{0}\left(q_{\mathrm{i}} g\right) J_{1}\left(u_{\mathrm{j}} g\right)-u_{\mathrm{j}} J_{1}\left(q_{\mathrm{i}} g\right) J_{0}\left(u_{\mathrm{j}} g\right)\right],
$$

$$
\lambda_{2}=-\frac{g J_{1}\left(u_{\mathrm{j}} g\right)}{v_{\mathrm{j}}^{2}-q_{\mathrm{i}}^{2}}\left[q_{\mathrm{i}} J_{0}\left(q_{\mathrm{i}} g\right) P_{1}\left(v_{\mathrm{j}} g\right)-v_{\mathrm{j}} J_{1}\left(q_{\mathrm{i}} g\right) P_{0}\left(v_{\mathrm{j}} g\right)\right] .
$$

The $\mathbf{D}, \mathbf{F}, \mathbf{G}, \mathbf{H}, \mathbf{G}^{\prime}, \mathbf{H}^{\prime}$ matrices have the same form as in the case of the E-cored coil, which was described in detail in [13], placed above the material without a hole. As to coefficients $\mathbf{C}_{\mathrm{i}}, \mathbf{B}_{\mathrm{i}}$, which are the solution of the system of the interface equations, they were normalized with respect to $\mathbf{B}_{7}$ and written down in the form of: $\mathbf{B}_{\mathrm{i} 7}=\mathbf{B}_{\mathrm{i}} / \mathbf{B}_{7}$, $\mathbf{C}_{\mathrm{i} 7}=\mathbf{C}_{\mathrm{i}} / \mathbf{B}_{7}$.

\section{RESULTS AND DISCUSSION}

The mathematical model created with the employment of the TREE method has been implemented in Matlab. In the calculations, the following assumptions have been taken: the number of summation terms $N s=115$ and the domain radius $b=12 r_{2}$. The average time necessary to calculate components of the coil impedance on a computer with an Intel i5-460M $2.53 \mathrm{GHz}$ processor and $6 \mathrm{~GB}$ of RAM was about 3 seconds. The employment of (13) allowed working out:

- coil impedance $Z=R+j X$ for conductive material with a hole,

- coil impedance $Z_{\mathrm{h}}=R_{\mathrm{h}}+j X_{\mathrm{h}}$ for material without a hole $(g=0)$

- coil impedance $Z_{0}=R_{0}+j X_{0}$ without the presence of a conductor $\left(\sigma_{6}=\sigma_{7}=0\right)$.

The correctness of the obtained results was verified by means of experimental measurements. For this purpose, a measurement system consisting of Agilent 4294A precision impedance analyzer and a two-layered plate of dimensions $200 \mathrm{~mm} \times 200 \mathrm{~mm}$ was created. The lower layer of the test object was a copper plate with a conductivity of $\sigma_{7}=7.7 \mathrm{MSm}^{-1}$ and thickness of $25 \mathrm{~mm}$. On the copper plate, a thin plate $\left(l_{2}-l_{1}=0.1 \mathrm{~mm}\right)$ made of aluminium alloy $5050\left(\sigma_{6}=28.1 \mathrm{MSm}^{-1}\right)$ was placed, and in its center there was a hole with a radius of $g=2 \mathrm{~mm}$. The measurements were made for 40 different frequency values in the range from $300 \mathrm{~Hz}$ to $20 \mathrm{kHz}$, and the parameters of the measurement system are presented in Table 1. 
Table 1. Parameters of the coil, core, and plate used in calculations.

\begin{tabular}{|c|c|c|}
\hline Inner column radius & $a_{1}$ & $1.5 \mathrm{~mm}$ \\
\hline Outer column radius & $a_{2}$ & $3.7 \mathrm{~mm}$ \\
\hline Inner core radius & $c_{1}$ & $7.7 \mathrm{~mm}$ \\
\hline Outer core radius & $c_{2}$ & $9.2 \mathrm{~mm}$ \\
\hline Inner core height & $d_{1}$ & $3.8 \mathrm{~mm}$ \\
\hline Outer core height & $d_{2}$ & $5.3 \mathrm{~mm}$ \\
\hline Inner coil radius & $r_{1}$ & $3.8 \mathrm{~mm}$ \\
\hline Outer coil radius & $r_{2}$ & $7.3 \mathrm{~mm}$ \\
\hline Offset & $h_{1}$ & $0.3 \mathrm{~mm}$ \\
\hline Parameter & $h_{2}$ & $3.7 \mathrm{~mm}$ \\
\hline Lift off & $l_{1}$ & $0.1 \mathrm{~mm}$ \\
\hline Radius of the domain & $b$ & $87.6 \mathrm{~mm}$ \\
\hline Relative permeability & $\mu_{\mathrm{f}}$ & 3000 \\
\hline Number of turns & $N$ & 646 \\
\hline Relative permeability & $\mu_{6}, \mu_{7}$ & 1 \\
\hline
\end{tabular}

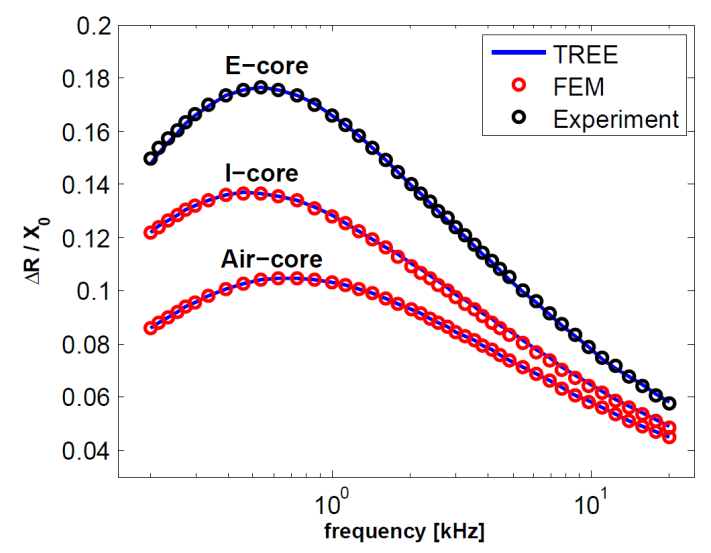

Fig.3. Normalized change in the coil resistance $\Delta R$ shown as a function of frequency.

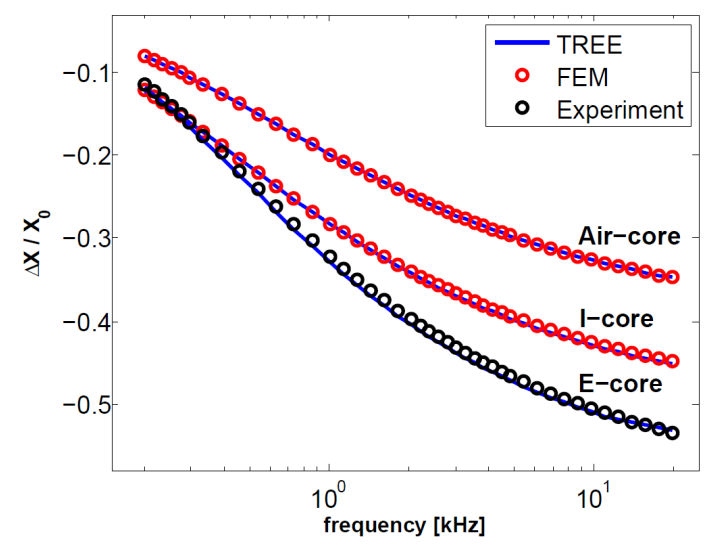

Fig.4. Normalized change in the coil reactance $\Delta X$ shown as a function of frequency.

The numerical verification of the calculation results was carried out using the finite element method. For this purpose, the COMSOL Multiphysics package was employed to create a model whose mesh contained 50468 triangular elements, 1098 edge elements, and 25 vertex elements.
Calculations of the changes of resistance $\Delta R=R-R_{0}$ and of reactance $\Delta X=X-X_{0}$ were also carried out for the I-cored coil and the air-cored coil. The impedance components of the I-cored coil were determined by substituting $c_{1}=c_{2}=a_{2}$ in (13), whereas for the air-cored coil $\mu_{\mathrm{f}}=1$ was assumed.

The achieved results were normalized with reference to $X_{0}$ and presented in Fig.3. and Fig.4. In comparison to experimental measurements, the maximum error of the changes in the components of impedance $\Delta Z$ obtained using the TREE method was $2.67 \%$ in the case of the real part and $2.86 \%$ in the case of the imaginary part. However, the differences in the results of the calculations made with the TREE and FEM methods did not exceed $1 \%$ for the E-cored coil, $1.82 \%$ for the I-cored coil and $0.5 \%$ for the air-cored coil.

The normalized changes of resistance $\Delta R$ shown in Fig.3. allow estimating the difference in the sensitivity of the measurement for three different types of coils. The largest changes in resistance were obtained at the frequency of approximately $500 \mathrm{~Hz}$. Obtaining much higher sensitivity of the measurement with the employment of the pot core in comparison with the I-core and the air-core probe is especially evident in the case of lower frequencies. The significance of this effect is much less considerable in the case of higher frequencies, when the depth of the penetration of eddy currents into the test material is shallower.

The changes in resistance $\Delta R_{\mathrm{h}}=R-R_{\mathrm{h}}$ and reactance $\Delta X_{\mathrm{h}}=X-X_{\mathrm{h}}$ resulting from the occurrence of the hole in the test workpiece are presented in Fig.5. The calculations were performed for bronze of the conductivity $5 \mathrm{MSm}^{-1}$, containing a surface hole with the radius $g=4.5 \mathrm{~mm}$ and depth $l_{2}-l_{1}=6 \mathrm{~mm}$. The maximum differences in the results achieved using the TREE and FEM methods were $2.77 \%$ for $R$ and $0.64 \%$ for $X$. In the case of the material without a hole, it was $1.35 \%$ for $R_{\mathrm{h}}$ and $0.46 \%$ for $X_{\mathrm{h}}$, respectively.

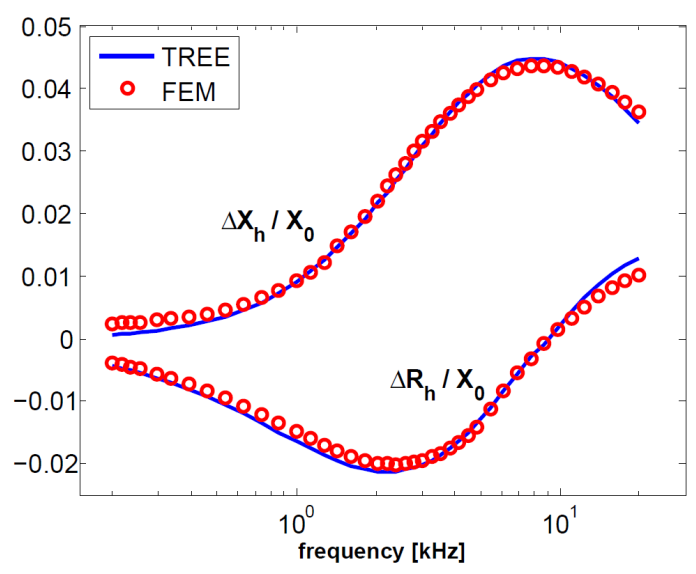

Fig.5. Normalized changes in the coil resistance $\Delta R_{\mathrm{h}}$ and reactance $\Delta X_{\mathrm{h}}$ as a function of frequency.

The method of eddy-current testing is applicable chiefly for the examination of non-ferromagnetic materials, but the solution presented herein makes it also possible to calculate components of coil impedance for ferromagnetic materials 
as well. With the assumption of the relative permeability $\mu_{6}=\mu_{7}=2$ calculations were made for the frequency $f=20 \mathrm{kHz}$ by means of the TREE and FEM methods. The deviations between components of the coil impedance were less than $0.8 \%$ for a material without a hole and $1.9 \%$ for a material with a hole. The hole in the ferromagnetic material under tests has led to the change of resistance by $8.5 \%$ and change of reactance by $1.5 \%$.

\section{CONCLUSIONS}

The mathematical model developed in this paper makes it possible to calculate the impedance of the E-cored coil placed above a two-layered conductive material with a surface hole of the radius $g$ and depth $l_{2}-l_{1}$. The presented final formulas may be implemented in any programming language or mathematical software, such as Matlab or Mathematica. The error control of the calculations is carried out through changing the values of the radius of domain $b$ and the number of summed terms Ns. The obtained results of the changes in resistance and reactance were verified by experimental measurements and the finite element method. In all of the cases, the calculation error did not exceed $3 \%$.

The proposed solution uses the pot core, which is the most commonly used in eddy current testing. By assuming the appropriate values of the input parameters, it is also possible to easily work out the final formulas for the air-cored coil or the I-cored coil. The carried out calculations enabled comparing the sensitivity of the coils in the cases of applying particular types of cores. The obtained changes of the components of impedance after the coil was brought closer to the conductive material with a hole were much bigger for the E-core than for the I-core and the air-core. It is due to the fact that the pot core increases the concentration of the magnetic flux in the test material, which ensures higher efficiency of detecting defects.

\section{REFERENCES}

[1] Theodoulidis, T., Wang, H., Tian., G.Y. (2012). Extension of a model for eddy current inspection of cracks to pulsed excitations. NDT\&E International, 47, 144-149.

[2] Qiu, X.B., Liu, L.L., Li, C.L., Wei, J.L., Wu, Y.F., Cui, X.C. (2014). Defect classification by pulsed eddycurrent technique based on power spectral density analysis combined with wavelet transform. IEEE Transactions on Magnetics, 50 (9), 6200708.

[3] Liu, B., Huang, P., Zeng, X., Li, Z. (2017). Hidden defect recognition based on the improved ensemble empirical decomposition method and pulsed eddy current testing. NDT\&E International, 86, 175-185.

[4] Lv, C., Tao, W., Lei, H., Jiang, Y., Zhao, H. (2015). Analytical modeling for the grating eddy current displacement sensors. Measurement Science Review, 15 (1), 44-51.

[5] Premel, D., Baussard, A. (2002). Eddy-current evaluation of three-dimensional flaws in flat conductive materials using a Bayesian approach. Inverse Problems, 18, 1873-1889.
[6] Durand, S., Cimrák, I., Sergeant, P., Abdallh, A. (2010). Analysis of a nondestructive evaluation technique for defect characterization in magnetic materials using local magnetic measurements. Mathematical Problems in Engineering, 2010, 574153.

[7] Kolyshkin, A. (2013). Solution of direct eddy current problems with cylindrical symmetry. In Proceedings of the International Conference on Application of Contemporary Non-destructive Testing in Engineering, 4-6 September 2013. Portoroz, Ljubljana, Slovenia: University of Ljubljana, 347-351.

[8] Koliskina, V. (2014). Semi-analytical solution of an eddy current problem for a conducting medium with a surface flaw. International Journal of Mathematical Models and Methods in Applied Sciences, 8, 473-480.

[9] Skarlatos, A., Theodoulidis, T. (2015). Calculation of the eddy-current flow around a cylindrical throughhole in a finite-thickness plate. IEEE Transactions on Magnetics, 51 (9), 6201507.

[10] Tytko, G., Dziczkowski, L. (2018). I-cored coil probe located above a conductive plate with a surface hole. Measurement Science Review, 18 (1), 7-12.

[11] Tytko, G., Dziczkowski, L. (2018). An analytical model of an I-cored coil located above a conductive material with a hole. European Physical Journal Applied Physics, 82 (2), 21001.

[12] Sakkaki, F., Bayani, H. (2012). Solution to the problem of E-cored coil above a layered half-space using the method of truncated region eigenfunction expansion. Journal of Applied Physics, 111 (7), $07 \mathrm{E} 717$.

[13] Tytko, G., Dziczkowski, L. (2015). E-cored coil with a circular air gap inside the core column used in eddy current testing. IEEE Transactions on Magnetics, 51 (9), 6201804.

[14] Theodoulidis, T.P., Bowler, J.R. (2004). The truncated region eigenfunction expansion method for the solution of boundary value problems in eddy current non-destructive evaluation. Review of Progress in Quantitative Nondestructive Evaluation, 24A, 403408.

[15] Theodoulidis, T.P., Kriezis, E.E. (2006). Eddy Current Canonical Problems (With Applications to Nondestructive Evaluation). Duluth (Georgia), USA: Tech Science Press.

[16] Tytko, G., Dawidowski, Ł. (2018). Locating complex eigenvalues for analytical eddy-current models used to detect flaws. Journal of Computational Physics, to be published.
Received August 6, 2018 Accepted March 05, 2019 